\title{
Average PER Performance Metrics of Iterative Successive Interference Cancellation
}

\author{
Francesc Molina, Student Member, IEEE, and Josep Sala-Álvarez, Senior Member, IEEE
}

\begin{abstract}
The average packet error rate (PER) and user-PER profiles of iterative successive interference cancellation (I-SIC) are analyzed for a proof-of-concept scenario where asymptotically many code-division-multiple-access users share the same channel encoder. To characterize the iterative decoding receiver, we propose a novel multivariate PER function evaluated over the consecutive signal-to-interference-plus-noise ratios (SINRs) experienced by each undecoded user. Analytic expressions for the user-energy and user-SINR profiles at every iteration are derived via warping operators on the user ordering that relate the asymptotic distribution of undecoded users at different iterations. Monte Carlo simulations using a chip-level I-SIC implementation assess the accuracy of the proposed user-limit analysis.
\end{abstract}

Index Terms-Iterative successive interference cancellation, multiple access, packet error rate, energy profile, warping

\section{INTRODUCTION}

$\mathbf{F}$ ORTHCOMING uplink scenarios with massive device connectivity pursue the introduction of interference cancellation (IC) receivers to wrestle with multiple access (MA) interference. SIC has been extensively analyzed as a multiuser decoding scheme that attains the corner points of the Gaussian MA channel capacity region [1]. Practical SIC, though, suffers performance degradation due to imperfect cancellation, error propagation and hardware impairments [2]. There is a potential for improvement using packet-oriented SIC as long as error control is performed at reception and I-SIC operates to tackle the multiple packet collisions [3]. In [3], [4], a number of ISIC systems have been simulated, proving the relevance of the decoder's PER characteristic to system performance.

Previous works have limited themselves to analyzing the average PER of a SIC receiver in which only a single iteration (one decoding attempt per user) is performed [5]. This strategy is reasonable when capacity-achieving encoders are employed since power control can be designed to decode all users at the first iteration [6]. In the massive user context, the use of short packets entails non-error free decoding for which I-SIC needs to iterate over still undecoded users (one or more decoding attempts per user) to enhance system performance.

This letter extends the non-iterative SIC in [5] to an I-SIC. We provide the analytic framework to analyze the I-SIC in the large-user regime, characterizing both the average PER and the user-PER profiles at every iteration as examples of

The authors are with the Department of Signal Theory and Communications of the Technical University of Catalonia (UPC), Barcelona (Spain). E-mails: \{francesc.molina, josep.sala\}@upc.edu. Work funded by project TEC201676409-C2-1-R (AEI/FEDER, UE) (WINTER): Spanish Ministry of Science, Innovation and Universities, and by the Catalan administration (AGAUR) under 2017 SGR 578 and FI-2018 supported by the Secretary for University and Research of the "Generalitat de Catalunya" and the European Social Fund. network- and user-centric metrics. A model is proposed that captures the essential statistical features of I-SIC decoding, and provides straightforward estimates of its performance metrics and its sensitivity to system parameters. To that aim, the multivariate PER characteristic quantifying the decoder's error rate under SINR gains over iterations is computed, while the average uncancelled energy is used to evaluate imperfect SIC cancellation. This differs from other works [3], [4] that consider the same PER function at each SIC iteration and heuristic criteria on the impact of imperfect cancellation.

Paper organization: Sec. II states the system model. Sec. III derives user-asymptotic equations. Sec. IV states PER bounds. Results and conclusions are offered in Secs. V and VI.

\section{SySTEM MODEL}

Let us consider a MA point receiving $n$-symbol packets from a large population of $K$ direct-sequence spread spectrum users. Packets $s_{k}[0 \leq m<n]$ comprise a known preamble, and data next to its cyclic redundancy check (CRC), both encoded via the same channel encoder. In a quasi-static flatfading channel where the user-channel gains are practically constant over the packet duration (a reasonable model for, e.g., some satellite communications scenarios in the framework of Enhanced Spread Spectrum ALOHA [3], [4], [7]), the received complex signal after down conversion is

$$
y(t)=\sum_{k=1}^{K} A[k] \sum_{m=0}^{n-1} s_{k}[m] c_{k, m}(t-\tau[k])+w(t),
$$

where $A[k] \triangleq\left(E_{\mathrm{r}}[k]\right)^{1 / 2} e^{j \varphi[k]}$ comprises the $k$-th user complex symbol energy $E_{\mathrm{r}}[k]$ and phase $\varphi[k] . c_{k, m}(t)$ is the $k$-th user spreading waveform of symbol $m, s_{k}[m]$, (according to the long code model in ref. [16] of [7]), $\tau[k]$ the $k$-th user delay, and $w(t)$ additive Gaussian noise.

\section{A. I-SIC operation}

The central MA node performs a packet-oriented I-SIC decoding strategy, such that, at every iteration $1 \leq i \leq I$, all unsuccessfully decoded users undergo a further decoding attempt as their SINR improves whenever other users are successfully decoded. Specifically, the first SIC run $i=1$ is performed in decreasing order of user-energies $E_{\mathrm{r}}[1 \leq k \leq K]$. Whenever a user is successfully decoded, i.e., after CRC, its packet is regenerated and canceled from the signal $y(t)$. Since ever fewer users remain undecoded after each new iteration, it is convenient to define user orderings (UO) at each iteration, as well as to establish indexing relationships between users that remain unsuccessfully decoded from iteration to iteration. 
Accordingly, we re-label the $i$-th UOs in agreement with the remaining users at the beginning of the $i$-th iteration, $K_{i}$, with $K_{1}=K$ the initial number of users, so that $1 \leq k_{i} \leq K_{i}$ denotes the $i$-th user index. Then, let

$$
\phi_{i, j} \quad: \quad k_{i} \longrightarrow k_{j}
$$

be a mapping between the $i$-th and the $j$-th $\mathrm{UO}$, that we use to relate user-energies between consecutive iterations, or w.r.t. the initial energy distribution $E_{\mathrm{r}}^{1}\left[k_{1}\right]=E_{\mathrm{r}}\left[k_{1}\right]$, using

$$
E_{\mathrm{r}}^{i+1}\left[k_{i+1}\right]=E_{\mathrm{r}}^{i}\left[\phi_{i+1, i}\left[k_{i+1}\right]\right] .
$$

\section{B. I-SIC model}

A simple and sufficiently accurate I-SIC model needs to capture some essential statistical features. In particular, statistical independence when decoding different users and the Gaussianity of MA interference after de-spreading (long code model, ref. [16] of [7]) are assumed. Therefore, we adopt a PER vs. SINR function after de-spreading that models an individual user's probability of incorrect decoding. One key aspect to incorporate, though, is that I-SIC will try to decode the same user again if all the previous attempts prove unsuccessful. Then, the success in decoding other users becomes relevant at each iteration in that: (i) if a sufficient fraction of users is decoded and canceled, the resulting signal is sufficiently modified in that a re-decoding attempt of any user can be assumed statistically independent from its previous attempts; (ii) if not enough users are decoded, the resulting signal does not change significantly, and re-decoding attempts of any user cannot be assumed independent from the previous ones.

Then, (i) differs with (ii) in that a user's SINR increases sufficiently w.r.t. its SINRs in past iterations: i.e., a univariate PER vs. SINR curve cannot model user decoding for all iterations. Thus, in agreement with Algorithm 1, we posit a multivariate function $\mathrm{PER}_{i}\left[\boldsymbol{\Gamma}^{i}\right]$ of the $1 \leq j \leq i$ SINRs after despreading an individual user, $\Gamma^{i} \triangleq\left[\Gamma^{1}, \Gamma^{2}, \ldots, \Gamma^{i}\right]$, to characterize channel decoding at the $i$-th SIC iteration. Specifically, the sequence of non-decreasing SINRs of user $k_{i}$ at $1 \leq j \leq i$ is

$$
\Gamma^{i}\left[k_{i}\right] \triangleq\left[\Gamma^{1}\left[\phi_{i, 1}\left[k_{i}\right]\right], \Gamma^{2}\left[\phi_{i, 2}\left[k_{i}\right]\right], \ldots, \Gamma^{i}\left[k_{i}\right]\right] .
$$

The SINR of user $k_{i}$ is $\Gamma^{i}\left[k_{i}\right]=E_{\mathrm{r}}^{i}\left[k_{i}\right] / N_{\mathrm{t}}^{i}\left[k_{i}\right]$, for which,

$$
\Gamma^{i}\left[k_{i}\right]=\frac{E_{\mathrm{r}}^{i}\left[k_{i}\right]}{N_{0}+\xi_{\mathrm{dcd}}^{i}+\frac{\theta}{N} \sum_{j<k_{i}} \epsilon_{j}\left(\Gamma^{i}[j]\right) E_{\mathrm{r}}^{i}[j]+\frac{\theta}{N} \sum_{j>k_{i}} E_{\mathrm{r}}^{i}[j]},
$$

with $N_{\mathrm{t}}^{i}\left[k_{i}\right]$ the $k_{i}$-th user's total noise plus interference term at the $i$-th iteration, comprising: the noise power spectral

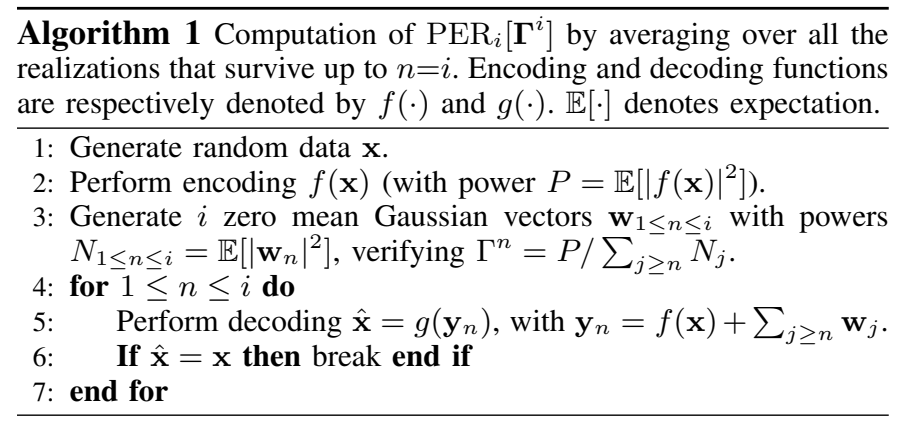

density (PSD) $N_{0}$, the aggregate residual interference $\xi_{\text {dcd }}^{i}$ after imperfect cancellation of successfully decoded users in preceding iterations, and summations $\sum_{j<k_{i}}(\cdot)$ and $\sum_{j>k_{i}}(\cdot)$ the interference from processed and unprocessed users in the current iteration. The effective inter-user decorrelation term $\left.\frac{\theta}{N}\right|_{0 \leq \theta \leq 1}$ models small inter-user time misalignments [8], with $N$ the spreading factor. $\epsilon\left(\boldsymbol{\Gamma}^{i}\right)$ is a binary random variable equal to 1 under incorrect CRC (with probability $\operatorname{PER}_{i}\left[\boldsymbol{\Gamma}^{i}\right]$ ), or, to the imperfect cancellation value $0 \leq \varepsilon\left(\Gamma^{i}\right) \leq 1$ otherwise.

Moreover, the term $\xi_{\mathrm{dcd}}^{i \geq 2}$ (with $\xi_{\mathrm{dcd}}^{1}=0$ ) is

$$
\xi_{\mathrm{dcd}}^{i \geq 2}=\frac{\theta}{N} \sum_{l=1}^{i-1} \sum_{j=1}^{K_{l}} \bar{\epsilon}_{j}\left(\boldsymbol{\Gamma}^{l}[j]\right) E_{\mathrm{r}}^{l}[j]
$$

where $\bar{\epsilon}\left(\boldsymbol{\Gamma}^{l}\right)$ denotes a binary random variable that takes the values: 0 (incorrect CRC, user still uncanceled) with probability $\mathrm{PER}_{l}\left[\boldsymbol{\Gamma}^{l}\right]$, or $\varepsilon\left(\Gamma^{l}\right)$ (correct CRC) otherwise.

\section{Average PER metrics}

Finally, in (7) we define the partial PER accounting for the average fraction of unsuccessfully decoded users at the $i$-th iteration, and in (8) the average PER after $i$ SIC iterations, computed as the product of partial PERs:

$$
\begin{aligned}
& \overline{\operatorname{per}}_{\mathrm{i}} \triangleq \frac{1}{K_{i}} \sum_{k_{i}=1}^{K_{i}} \operatorname{PER}_{i}\left[\Gamma^{i}\left[k_{i}\right]\right], \\
& \overline{\mathrm{per}}_{\mathrm{all}, \mathrm{i}} \triangleq \prod_{j=1}^{i} \overline{\operatorname{per}}_{j} .
\end{aligned}
$$

\section{USER-ASYMPTOTIC EXPRESSIONS}

This Section derives the user-limit expressions of the prior system model. In the user-asymptotic case, the continuous user indices $t_{i} \triangleq \lim _{K_{i} \rightarrow+\infty} k_{i} / K_{i}$ are defined, with the traffic load $\alpha_{i} \triangleq K_{i} / N$ at iteration $i$ fixed when $N \rightarrow+\infty$, as is exposed after equation (13) and validated later on. Moreover, $\Gamma^{i}\left[k_{i}\right], E_{\mathrm{r}}^{i}\left[k_{i}\right], \phi_{i, j}\left[k_{i}\right]$ are converted to the continuous functions $\Gamma^{i}\left(t_{i}\right), E_{\mathrm{r}}^{i}\left(t_{i}\right), \phi_{i, j}\left(t_{i}\right)$ also referred to as profiles, where, e.g., $\Gamma^{i}\left[\alpha_{i} t_{i} N\right] \rightarrow \Gamma^{i}\left(t_{i}\right)$, with domain $0 \leq t_{i} \leq 1$.

\section{A. User-asymptotic ordering}

Firstly, the following properties on the composition of the mappings in (2) are derived, with inverse mapping $\phi_{i, j}^{-1}=\phi_{j, i}$ :

$$
\begin{aligned}
& \text { Property 1: } \phi_{i, j<i}=\phi_{j+1, j} \circ \phi_{j+2, j+1} \circ \cdots \circ \phi_{i, i-1} \\
& \text { Property 2: } \phi_{i, j>i}=\phi_{j-1, j} \circ \phi_{j-2, j-1} \circ \cdots \circ \phi_{i, i+1}
\end{aligned}
$$

Secondly, as a consequence of the employed I-SIC scheme, at the end of each $i$, and over every interval $\left[t_{i}, t_{i}+\mathrm{d} t_{i}\right]$, a fraction $\operatorname{PER}\left[\boldsymbol{\Gamma}^{i}\left(t_{i}\right)\right] \mathrm{d} t_{i}$ of users remains undecoded for subsequent SIC iterations. Then, the relationship between the $i$-th and the $(i+1)$-th UOs is given by $t_{i+1}=\phi_{i, i+1}\left(t_{i}\right)$, with

$$
\phi_{i, i+1}\left(t_{i}\right) \triangleq \int_{0}^{t_{i}} \operatorname{PER}_{i}\left[\boldsymbol{\Gamma}^{i}(\tau)\right] \mathrm{d} \tau / \int_{0}^{1} \operatorname{PER}_{i}\left[\boldsymbol{\Gamma}^{i}(\tau)\right] \mathrm{d} \tau
$$

where, and without loss of generality, the denominator is introduced as a normalization term, such that, (11) constitutes a bijective mapping from $[0,1]$ onto itself, and thus, it performs a warping of the user-variable $t_{i}$. Hence (3) is turned to

$$
E_{\mathrm{r}}^{i+1}\left(t_{i+1}\right)=E_{\mathrm{r}}^{i}\left(\phi_{i+1, i}\left(t_{i+1}\right)\right) .
$$




\section{B. User-asymptotic SINR profile}

We first let (5) go to the user-limit. We multiply and divide each summation by $K_{i}$, and we set: (i) $\alpha_{i} \rightarrow K_{i} / N$; and (ii) $\mathrm{d} \tau \rightarrow 1 / K_{i}$. We get $\Gamma^{i}\left(t_{i}\right)=E_{\mathrm{r}}^{i}\left(t_{i}\right) / N_{\mathrm{t}}^{i}\left(t_{i}\right)$, or equivalently:

$$
\Gamma^{i}\left(t_{i}\right)=\frac{E_{\mathrm{r}}^{i}\left(t_{i}\right)}{N_{0}^{i}+\alpha_{i} \theta \int_{0}^{t_{i}} r_{i}\left(\Gamma^{i}(\tau)\right) E_{\mathrm{r}}^{i}(\tau) \mathrm{d} \tau+\alpha_{i} \theta I_{i}\left(t_{i}\right)},
$$

with $N_{\mathrm{t}}^{i}\left(t_{i}\right)$ the denominator in (13), $N_{0}^{i} \triangleq N_{0}+\xi_{\mathrm{dcd}}^{i}$ and $\alpha_{i} \triangleq \alpha_{i-1} \overline{\operatorname{per}}_{i-1}$, respectively, the noise plus interference from decoded users and the traffic load at the beginning of the $i$-th iteration, $I_{i}(t) \triangleq \int_{t}^{1} E_{\mathrm{r}}^{i}(\tau) \mathrm{d} \tau$ the integrated $i$ th energy profile, and $r_{i}\left(\boldsymbol{\Gamma}^{i}\right) \triangleq 1-\left(1-\varepsilon\left(\Gamma^{i}\right)\right) \operatorname{PSR}_{i}\left[\boldsymbol{\Gamma}^{i}\right]$ defined from $\operatorname{PSR}_{i}\left[\boldsymbol{\Gamma}^{i}\right] \triangleq 1-\operatorname{PER}_{i}\left[\boldsymbol{\Gamma}^{i}\right]$, the packet success rate (PSR) function. Moreover, (6) asymptotically converges to the following deterministic expression

$$
\xi_{\mathrm{dcd}}^{i \geq 2}=\sum_{l=1}^{i-1} \alpha_{l} \theta \int_{0}^{1} \varepsilon\left(\Gamma^{l}(\tau)\right) \operatorname{PSR}_{l}\left[\boldsymbol{\Gamma}^{l}(\tau)\right] E_{\mathrm{r}}^{l}(\tau) \mathrm{d} \tau .
$$

From [8] and for the given $E_{\mathrm{r}}^{i}\left(t_{i}\right)$ in (12), the $i$-th asymptotic SINR profile $\Gamma^{i}\left(t_{i}\right)$ can be determined from an ordinary differential equation (ODE). Specifically, we apply the gradient $\nabla_{t_{i}}$ under variable $t_{i}$ to $N_{\mathrm{t}}^{i}\left(t_{i}\right)=E_{\mathrm{r}}^{i}\left(t_{i}\right) / \Gamma^{i}\left(t_{i}\right)$ in (13). Finally, dividing at both sides by $N_{\mathrm{t}}^{i}\left(t_{i}\right)$ and arranging terms, we obtain, for a known $E_{\mathrm{r}}^{i}\left(t_{i}\right)$, the following ODE in $\Gamma^{i}\left(t_{i}\right)$ :

$$
\nabla_{t_{i}} \log N_{\mathrm{t}}^{i}\left(t_{i}\right)=-\alpha_{i} \Phi_{i}\left[\boldsymbol{\Gamma}^{i}\left(t_{i}\right)\right],
$$

with $\Phi_{i}\left[\boldsymbol{\Gamma}^{i}\right] \triangleq \theta\left(1-\varepsilon\left(\Gamma^{i}\right)\right) \Gamma^{i} \operatorname{PSR}_{i}\left[\boldsymbol{\Gamma}^{i}\right]$ a known function, and $\log$ the natural logarithm. The ODE in (15) satisfies the boundaries $N_{\mathrm{t}}^{1}(0)=N_{0}+\alpha_{1} \theta I_{1}(0)$ and $N_{\mathrm{t}}^{i \geq 2}(0)=N_{\mathrm{t}}^{i-1}(1)$ for the corresponding solution $\Gamma^{i}\left(t_{i}\right)$, which together with (11) and (12), constitute a chain of ODEs for $1 \leq i \leq I$. Then, integrating (15), $N_{\mathrm{t}}^{i}\left(t_{i}\right)$ can be computed from $\boldsymbol{\Gamma}^{i}\left(t_{i}\right)$ as

$$
N_{\mathrm{t}}^{i}\left(t_{i}\right)=N_{\mathrm{t}}^{i-1}(1) \exp \left(-\alpha_{i} \int_{0}^{t_{i}} \Phi_{i}\left[\boldsymbol{\Gamma}^{i}(\tau)\right] \mathrm{d} \tau\right)
$$

to generate $N_{\mathrm{t}}^{i+1}(0)=N_{\mathrm{t}}^{i}(1)$ for the next iteration. Thus, the SINR vector (4) expressed over variable $t_{i}$ is

$$
\Gamma^{i}\left(t_{i}\right) \triangleq\left[\Gamma^{1}\left(\phi_{i, 1}\left(t_{i}\right)\right), \Gamma^{2}\left(\phi_{i, 2}\left(t_{i}\right)\right), \ldots, \Gamma^{i}\left(t_{i}\right)\right],
$$

with $\Gamma^{k}\left(\phi_{i, k}\left(t_{i}\right)\right)=E_{\mathrm{r}}^{k}\left(\phi_{i, k}\left(t_{i}\right)\right) / N_{\mathrm{t}}^{k}\left(\phi_{i, k}\left(t_{i}\right)\right)$ being the $k$-th element of (17). Now, using (12) and the property (9) with $j=1$ for the energies in the numerators, $E_{\mathrm{r}}^{j}\left(\phi_{i, j}\left(t_{i}\right)\right)$ can be expressed as a function of the initial energy profile as $E_{\mathrm{r}}^{j}\left(\phi_{i, j}\left(t_{i}\right)\right)=E_{\mathrm{r}}^{1}\left(\phi_{i, 1}\left(t_{i}\right)\right)$. Therefore, (17) yields

$$
\boldsymbol{\Gamma}^{i}\left(t_{i}\right)=\left[\frac{E_{\mathrm{r}}^{1}\left(\phi_{i, 1}\left(t_{i}\right)\right)}{N_{\mathrm{t}}^{1}\left(\phi_{i, 1}\left(t_{i}\right)\right)}, \frac{E_{\mathrm{r}}^{1}\left(\phi_{i, 1}\left(t_{i}\right)\right)}{N_{\mathrm{t}}^{2}\left(\phi_{i, 2}\left(t_{i}\right)\right)}, \ldots, \frac{E_{\mathrm{r}}^{1}\left(\phi_{i, 1}\left(t_{i}\right)\right)}{N_{\mathrm{t}}^{i}\left(t_{i}\right)}\right] .
$$

\section{User-asymptotic average PER and user-PER profile}

We derive next our main result: the user-limit average and user-PER profile of the I-SIC receiver over the initial UO $t_{1}$.

1) Step 1: The partial PER in (7) asymptotically tends to

$$
\overline{\operatorname{per}}_{\mathrm{i}}=\int_{0}^{1} \operatorname{PER}_{i}\left[\boldsymbol{\Gamma}^{i}\left(t_{i}\right)\right] \mathrm{d} t_{i} .
$$

The average PER (8), the product of partial PERs, can still be simplified using the warping operators defined herein.
Using (18) in (19) and the change of variable $\lambda \triangleq \phi_{i, 1}\left(t_{i}\right)$ with $t_{i}=\phi_{1, i}(\lambda)$ and $\mathrm{d} t_{i}=\phi_{1, i}^{\prime}(\lambda) \mathrm{d} \lambda$, we get (20) in terms of $\mathbf{G}^{i}\left(t_{1}\right) \triangleq \boldsymbol{\Gamma}^{i}\left(\phi_{1, i}\left(t_{1}\right)\right)$ defined in (21):

$$
\begin{aligned}
\overline{\operatorname{per}}_{\mathrm{i}} & =\int_{0}^{1} \operatorname{PER}_{i}\left[\mathbf{G}^{i}(\lambda)\right] \phi_{1, i}^{\prime}(\lambda) \mathrm{d} \lambda, \\
\mathbf{G}^{i}\left(t_{1}\right) & =\left[\frac{E_{\mathrm{r}}^{1}\left(t_{1}\right)}{N_{\mathrm{t}}^{1}\left(t_{1}\right)}, \frac{E_{\mathrm{r}}^{1}\left(t_{1}\right)}{N_{\mathrm{t}}^{2}\left(\phi_{1,2}\left(t_{1}\right)\right)}, \ldots, \frac{E_{\mathrm{r}}^{1}\left(t_{1}\right)}{N_{\mathrm{t}}^{i}\left(\phi_{1, i}\left(t_{1}\right)\right)}\right],
\end{aligned}
$$

where for the arguments of $N_{\mathrm{t}}^{l \leq i}\left(\phi_{i, l}\left(\phi_{1, i}\right)(\lambda)\right)$ we have used the property (10): $\left(\phi_{i, l} \circ \phi_{1, i}\right)(\lambda)=\phi_{1, l}(\lambda)$. Moreover, the term $\phi_{1, i}(\lambda)$ in (21) equals $\left(\phi_{i-1, i} \circ \phi_{1, i-1}\right)(\lambda)$, and thus, the derivative $\phi_{1, i}^{\prime}(\lambda)$ can be recursively expressed as

$$
\phi_{1, i}^{\prime}(\lambda)=\operatorname{PER}_{i-1}\left[\mathbf{G}^{i-1}(\lambda)\right] \cdot \phi_{1, i-1}^{\prime}(\lambda) \cdot\left(\overline{\operatorname{per}}_{\mathrm{i}-1}\right)^{-1} .
$$

2) Step 2: The average PER (8) is now obtained: firstly, substituting (20), and secondly, by recursive application of (22). This yields (23), where its integrand corresponds to the user-PER profile after $i$ SIC iterations defined in (24):

$$
\begin{aligned}
\overline{\operatorname{per}}_{\mathrm{all}, \mathrm{i}} & =\int_{0}^{1} \prod_{j=1}^{i} \operatorname{PER}_{j}\left[\mathbf{G}^{j}(\lambda)\right] \mathrm{d} \lambda, \\
\operatorname{PER}_{i}\left(t_{1}\right) & =\prod_{j=1}^{i} \operatorname{PER}_{j}\left[\mathbf{G}^{j}\left(t_{1}\right)\right] .
\end{aligned}
$$

\section{A Bound on the USER-ERror PRobability}

The computational complexity for obtaining $\operatorname{PER}_{I}\left[\boldsymbol{\Gamma}^{I}\right]$ is exponential in $I$. Thus, it is of interest to consider a simple upper bound for the user-error probability after $I$ iterations

$$
P_{I} \triangleq \prod_{i=1}^{I} \operatorname{PER}_{i}\left[\boldsymbol{\Gamma}^{i}\right],
$$

with $I=2$ a case of particular interest since Section $\mathrm{V}$ evaluates the accuracy of the approximation $\operatorname{PER}_{I}\left[\Gamma^{1}, \cdots, \Gamma^{I}\right] \simeq$ $\mathrm{PER}_{2}\left[\Gamma^{I-1}, \Gamma^{I}\right]$ and the related upper bound in (26). Let $\mathcal{U}$ denote a set of realizations $u_{l}=\left\{\mathbf{x}, \mathbf{w}_{1 \leq n \leq I}\right\}_{l}$ of Algorithm 1, for which one packet may undergo up to $I$ decoding attempts. To obtain an upper-bound over (25), we compare two policies at the same $\Gamma^{I}=\Gamma^{*}$, with $\Gamma^{*}$ known. For conciseness, the following rationale is outlined for $I \leq 2$ :

(i) $I=1$ iteration: $\mathcal{U}$ is partitioned into the disjoint subsets $\mathcal{U}_{\mathrm{ok}}^{1}$ and $\mathcal{U}_{\mathrm{ko}}^{1}$, respectively denoting the subsets of $u_{l} \in \mathcal{U}$ that succeed $(\mathrm{OK})$ and fail $(\mathrm{KO})$ under $\Gamma^{1}=\Gamma^{*}$.

(ii) $I=2$ iterations: At $i=1, \mathcal{U}$ is partitioned into the subsets $\mathcal{U}_{\mathrm{ok}}^{2}$ and $\mathcal{U}_{\mathrm{ko}}^{2}$ at $\Gamma^{1} \leq \Gamma^{*}$. In this case, only those $u_{l} \in \mathcal{U}_{\mathrm{ko}}^{2}$ undergo a further attempt. At $i=2, \Gamma^{2}=\Gamma^{*}$, and $\mathcal{U}_{\mathrm{ko}}^{2}$ is partitioned into $\mathcal{U}_{\mathrm{ko}, \mathrm{ok}}^{2}(\mathrm{OK})$ and $\mathcal{U}_{\mathrm{ko}, \mathrm{ko}}^{2}(\mathrm{KO})$.

In each case, the user-error probabilities (25) are, respectively: (i) $\operatorname{PER}_{1}\left[\Gamma^{*}\right]=\left|\mathcal{U}_{\mathrm{ko}}^{1}\right| /|\mathcal{U}|$, with $|\cdot|$ the cardinality operator; and (ii) $\operatorname{PER}_{1}\left[\Gamma^{1}\right] \mathrm{PER}_{2}\left[\Gamma^{1}, \Gamma^{*}\right]=\left|\mathcal{U}_{\mathrm{ko}, \mathrm{ko}}^{2}\right| /|\mathcal{U}| . \mathcal{U}_{\mathrm{ko}, \mathrm{ko}}^{2}$ contains the set of $u_{l} \in \mathcal{U}$ that fail at both $\Gamma^{1} \leq \Gamma^{*}$ and $\Gamma^{2}=\Gamma^{*}$, which is contained in $\mathcal{U}_{\mathrm{ko}}^{1}$, the set of $u_{l} \in \mathcal{U}$ that fail at $\Gamma^{1}=\Gamma^{*}$. Then, $\mathcal{U}_{\mathrm{ko}, \mathrm{ko}}^{2} \subseteq \mathcal{U}_{\mathrm{ko}}^{1}$, which implies $\left|\mathcal{U}_{\mathrm{ko}, \mathrm{ko}}^{2}\right| \leq\left|\mathcal{U}_{\mathrm{ko}}^{1}\right|$, or, equivalently, the following bound used in Section $\mathrm{V}$,

$$
\operatorname{PER}_{1}\left[\Gamma^{1}\right] \mathrm{PER}_{2}\left[\Gamma^{1}, \Gamma^{*}\right] \leq \operatorname{PER}_{1}\left[\Gamma^{*}\right] .
$$

We remark the previous bound is only valid for $\Gamma^{1} \leq \Gamma^{*}$. Otherwise, for $\Gamma^{1} \geq \Gamma^{*}, \mathrm{PER}_{2}\left[\Gamma^{1}, \Gamma^{*}\right]=1$. By a similar procedure, expression (26) can be generalized to $P_{I} \leq \mathrm{PER}_{1}\left[\Gamma^{I}\right]$ for $I \geq 3$. The proof is omitted for lack of space. 


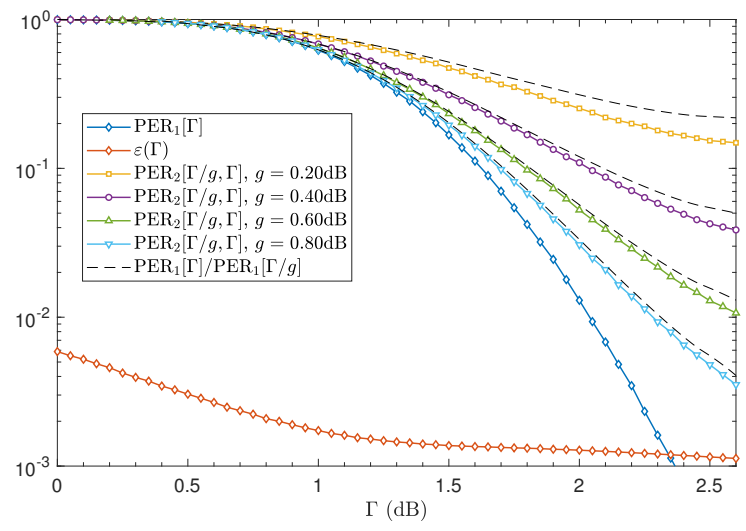

Fig. 1. $\operatorname{PER}_{1}[\Gamma]$ and $\varepsilon(\Gamma)$ (blue and red diamond) vs. $\Gamma$. $\mathrm{PER}_{2}[\Gamma / g, \Gamma]$ (not diamond) and $\mathrm{PER}_{1}[\Gamma] / \operatorname{PER}_{1}[\Gamma / g]$ (dashed) vs. $\Gamma$ for several $g$ values.

\section{Numerical Results}

For simulations, we adopt, in compliance with ETSI [9], the standardized DVB-RCS turbo code of rate $1 / 2$ with QPSK and a shaping pulse of roll-off 0.35 . Packets contain a preamble of 48 symbols followed by 440 payload symbols. At reception, $I=3$ SIC iterations are performed.

To model channel decoding, we use $\mathrm{PER}_{1}\left[\Gamma^{1}\right]$ at $i=1$ and the two-variate $\mathrm{PER}_{2}\left[\Gamma^{1}, \Gamma^{2}\right]$ at $i=2$. The multivariate PER at $i=3$ is approximated by $\mathrm{PER}_{3}\left[\Gamma^{1}, \Gamma^{2}, \Gamma^{3}\right] \approx \mathrm{PER}_{2}\left[\Gamma^{2}, \Gamma^{3}\right]$. Fig. 1 shows $\operatorname{PER}_{1}[\Gamma], \varepsilon(\Gamma)$ and $\operatorname{PER}_{2}[\Gamma / g, \Gamma]$ for some $g$. As shown, at low PER values $\left(<10^{-1}\right) \mathrm{PER}_{2}[\Gamma / g, \Gamma]$ approaches the univariate $\operatorname{PER}_{1}[\Gamma]$ as $g$ grows, since such increment makes the decoder see increasingly different noise realizations between $i=1,2$. Moreover, the upper bound $\mathrm{PER}_{2}[\Gamma / g, \Gamma] \leq \frac{\mathrm{PER}_{1}[\Gamma]}{\mathrm{PER}_{1}[\Gamma / g]}$ (dashed) taken from (26) results in a visible, yet small gap for $g<0.4 \mathrm{~dB}$, mitigated as $g$ increases.

In Fig. 2, we compare the empirical PER profiles from a chip-level SIC implementation with their asymptotics (24). Regarding simulation parameters, the chip rate is fixed to 3.84 Mcps. The traffic load is set to $\alpha=\frac{K}{N}=0.5$ and the factor $\theta$ to 1 . Therefore, $K=64,128,256$ users are evaluated under the corresponding symbol rates $15,7.5$ and 5 kbaud. At reception, we consider users uniformly sampled from the two-piecewise symbol energy over noise PSD $\left(E_{\mathrm{s}} / N_{0}\right)$ profile

$$
\frac{E_{\mathrm{r}}(t)}{N_{0}}=\left\{\begin{array}{llc}
-1.68 t+2.75 & \text { if } & 0 \leq t<0.8 \\
1.41 & \text { if } & 0.8 \leq t \leq 1
\end{array} .\right.
$$

The convergence of the empirical PER to their asymptotics is subject to having enough users at $i \geq 2$ so that: (i) $\alpha_{i}$ is kept constant; and (ii) $E_{\mathrm{r}}^{i}\left(t_{i}\right)$ can be deterministically obtained from (12). The trend of asymptotic profiles (solid) is well predicted by empirical PER (markers) when $N \geq 256$. Empirical profiles approach user-asymptotic ones as $N$ increases. Mismatching errors due to finite-user effects appear at those $t$-values associated with low PER values since hypothesis (ii) weakens. The third iteration, although $\Gamma^{1}$-independent, performs well. The upper bounds (dashed) are found to be an accurate approximation subject to less computational cost.

The $E_{\mathrm{s}} / N_{0}$ profiles at every iteration are depicted in Fig. 2 's inset. They are shown to converge to uniform profiles over SIC iterations, since stronger users are demodulated with lower PER and subsequent SIC iterations handle the weakest users.

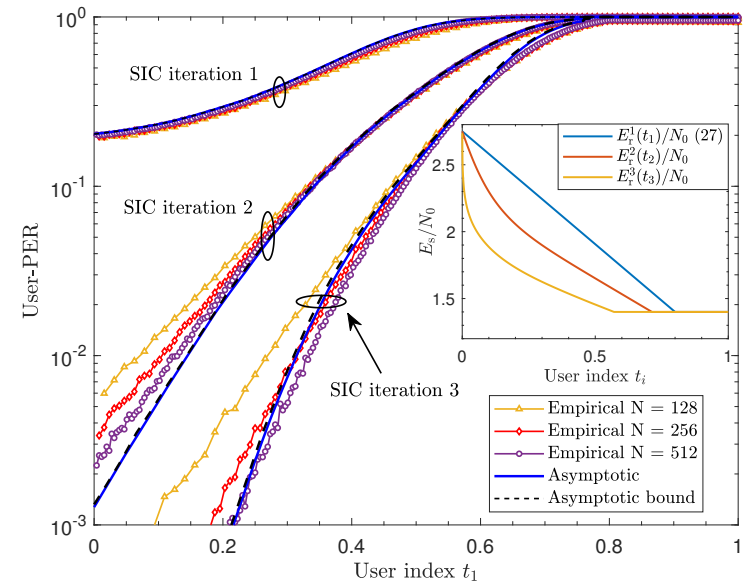

Fig. 2. Asymptotic (solid) and empirical (marker) user-PER profiles at each iteration for different spreading gains. Empirical user-PER profiles have been obtained by averaging $10^{5}$ Monte Carlo runs. Dashed lines denote the userasymptotic PER profiles using: $\mathrm{PER}_{2}\left[\Gamma^{1}, \Gamma^{2}\right]=\mathrm{PER}_{1}\left[\Gamma^{2}\right] / \mathrm{PER}_{1}\left[\Gamma^{1}\right]$ for $i=2$, and $\operatorname{PER}_{2}\left[\Gamma^{2}, \Gamma^{3}\right]=\operatorname{PER}_{1}\left[\Gamma^{3}\right] / \operatorname{PER}_{1}\left[\Gamma^{2}\right]$ for $i=3$. Nested figure shows the user-asymptotic $E_{\mathrm{S}} / N_{0}$ profiles at SIC iterations $1 \leq i \leq 3$.

\section{CONCLUSIONS}

The average PER and user-PER of I-SIC have been derived for an uplink setting where a large number of spread spectrum users employ the same encoder. A multivariate PER function has been adopted, instead of the univariate PER in [3], [5], [8], to capture the decoding behavior of users through SIC iterations. The user-asymptotic energy and SINR profiles at every SIC iteration have been obtained by solving a chain of differential equations. An upper bound on the stated multivariate PER has been proposed. Simulations using a chip-level SIC implementation have assessed, for a sufficiently high number of users, the success of the undertaken analysis, showing that a two-variate PER over the two last SINR arguments can model I-SIC accurately under $2 \leq I \leq 3$ iterations.

\section{REFERENCES}

[1] X. Zhang and M. Haenggi, "The performance of successive interference cancellation in random wireless networks," IEEE Trans. Inf. Theory, vol. 60, no. 10, pp. 6368-6388, Oct. 2014.

[2] N. I. Miridakis and T. A. Tsiftsis, "On the joint impact of hardware impairments and imperfect CSI on successive decoding," IEEE Trans. Veh. Technol., vol. 66, no. 6, pp. 4810-4822, Jun. 2017.

[3] F. Collard and R. De Gaudenzi, "On the optimum packet power distribution for spread ALOHA packet detectors with iterative successive interference cancelation," IEEE Trans. Wireless Commun., vol. 13, no. 12, pp. 6783-6794, Dec. 2014.

[4] R. De Gaudenzi, O. Del Río Herrero, and G. Gallinaro, "Enhanced spread ALOHA physical layer design and performance," Int. Journal on Satellite Communications and Networking, vol. 32, no. 6, pp. 457-473, Nov. 2014.

[5] J. Sala, J. Villares, and F. Rey, "Asymptotic and finite user PER analysis of successive interference cancellation for DS-CDMA," IEEE Commun. Lett., vol. 15, no. 11, pp. 1145-1147, Nov. 2011.

[6] D. V. Djonin and V. K. Bhargava, "Asymptotic analysis of the conventional decision feedback receiver in fading channels," IEEE Trans. Wireless Commun., vol. 2, no. 5, pp. 1066-1078, Sep. 2003.

[7] J. Sala-Álvarez, F. Rey, J. Villares, and F. Molina, "Minimum PER user-energy profile for massive SIC receivers under an average energy constraint," in 2017 IEEE 18th Int. Workshop on Signal Processing Advances in Wireless Communications (SPAWC), Jul. 2017, pp. 1-6.

[8] J. Sala, F. Rey, and J. Villares, "Asymptotically optimum energy profile for successive interference cancellation in DS-CDMA under a power unbalance constraint," IEEE Commun. Lett., vol. 16, no. 2, pp. 172-175, Feb. 2012.

[9] EN ETSI, "Digital video broadcasting (DVB); interaction channel for satellite distribution systems," ETSI EN 301790 (V1.4.1), 2005. 